\title{
Leptin and adiponectin DNA methylation levels in adipose tissues and blood cells are associated with BMI, waist girth and LDL-cholesterol levels in severely obese men and women
}

\author{
Andrée-Anne Houde ${ }^{1,2}$, Cécilia Légaré ${ }^{1,2}$, Simon Biron ${ }^{3,4}$, Odette Lescelleur ${ }^{3,4}$, Laurent Biertho ${ }^{3,4}$, Simon Marceau ${ }^{3,4}$,
} André Tchernof ${ }^{3,5,6}$, Marie-Claude Vohl ${ }^{5,6,7}$, Marie-France Hivert ${ }^{8,9,10}$ and Luigi Bouchard ${ }^{1,2^{*}}$

\begin{abstract}
Background: Leptin (LEP) and adiponectin (ADIPOQ) genes encode adipokines that are mainly secreted by adipose tissues, involved in energy balance and suspected to play a role in the pathways linking adiposity to impaired glucose and insulin homeostasis. We have thus hypothesized that LEP and ADIPOQ DNA methylation changes might be involved in obesity development and its related complications. The objective of this study was to assess whether LEP and ADIPOQ DNA methylation levels measured in subcutaneous (SAT) and visceral adipose tissues (VAT) are associated with anthropometric measures and metabolic profile in severely obese men and women. These analyses were repeated with DNA methylation profiles from blood cells obtained from the same individuals to determine whether they showed similarities.

Methods: Paired SAT, VAT and blood samples were obtained from 73 severely obese patients undergoing a bioliopancreatic diversion with duodenal switch. LEP and ADIPOQ DNA methylation and mRNA levels were quantified using bisulfite-pyrosequencing and qRT-PCR respectively. Pearson's correlation coefficients were computed to determine the associations between $\angle E P$ and ADIPOQ DNA methylation levels, anthropometric measures and metabolic profile.

Results: DNA methylation levels at the ADIPOQ gene locus in SAT was positively associated with BMI and waist girth whereas LEP DNA methylation levels in blood cells were negatively associated with body mass index (BMI). Fasting LDL-C levels were found to be positively correlated with DNA methylation levels at LEP-CpG11 and -CpG17 in blood and SAT and with ADIPOQ DNA methylation levels in SAT (CPGE1 and CPGE3) and VAT (CPGE1).

Conclusions: These results confirm that $\angle E P$ and $A D I P O Q$ epigenetic profiles are associated with obesity. We also report associations between LDL-C levels and both $L E P$ and ADIPOQ DNA methylation levels suggesting that LDL-C might regulate their epigenetic profiles in adipose tissues. Furthermore, similar correlations were observed between LDL-C and LEP blood DNA methylation levels suggesting a common regulatory pathway of DNA methylation in both adipose tissues and blood.
\end{abstract}

Keywords: Subcutaneous adipose tissue, Visceral adipose tissue, Epigenetic, Metabolic complications

\footnotetext{
* Correspondence: luigi.bouchard@usherbrooke.ca

'Department of Biochemistry, Université de Sherbrooke, Sherbrooke, QC,

Canada

2ECOGENE-21 and Clinical Research Center and Lipid Clinic, Chicoutimi

Hospital, Saguenay, QC, Canada

Full list of author information is available at the end of the article
} 


\section{Background}

The incidence of obesity and its related disorders (dyslipidemia, hypertension, type 2 diabetes and cardiovascular diseases) has been constantly increasing in the last few decades leading to a global obesity epidemic [1,2]. Although the heritability estimates for obesity range between 6\%-85\% depending on the trait assessed [3], the obesity-related genetic variants identified have so far explained less than $2 \%$ of the heritability of obesity $[4,5]$. Hence, it is unlikely that the current obesity epidemic is solely caused by genetic variations. The interactions between the gene variants and the components of our obesogenic environment are very likely contributing to the increasing obesity rates.

Genes are known to adapt to the environment through epigenetic modifications [6,7] among other mechanisms. Epigenetics refers to the molecular mechanisms regulating gene expression without affecting the DNA sequence [8]. DNA methylation, the most understood epigenetic mark, primarily occurs on the cytosine upstream of a guanine (dinucleotide $\mathrm{CpG}$ ) and is catalyzed by the DNA methyltransferases (DNMTs) [9]. The methylation of cytosines in a CpG context has been shown to be sensitive to environmental stimuli including in utero [10-12] and post-natal environmental conditions $[13,14]$.

Epigenetic modifications contribute to the pathogenesis of obesity and its obesity-related metabolic complications. Indeed, studies have reported that DNA methylation levels at candidate gene loci related to obesity and metabolic diseases are impaired in blood and adipose tissues of obese patients $[15,16]$ and in low weight loss responder to diet and exercise interventions [17-20]. The leptin $(L E P)$ and adiponectin $(A D I P O Q)$ genes are probably those that have warranted the most attention so far. These genes encode for leptin and adiponectin proteins, which are mainly synthesized and secreted by the adipocytes. Leptin plasma concentrations are increased in obese subjects (leptin resistance is suspected) and has both anorexigenic and proinflammatory properties [21,22], whereas adiponectin improves insulin sensitivity, exerts anti-inflammatory actions and its secretion is significantly reduced in obesity [21,22].

We have previously reported that maternal hyperglycaemia ( $2 \mathrm{~h}$ post-oral glucose tolerance test (OGTT)) during the second trimester of pregnancy is associated with decreased placental DNA methylation levels at $L E P$ [23] and ADIPOQ [24] gene loci suggesting that epigenetic adaptations could be involved in fetal metabolic programming and increase newborn lifelong susceptibility to obesity and metabolic disorders. Other groups have reported that whole blood $L E P$ DNA methylation levels are negatively associated with birth weight and child BMI at 17 months [25]. In addition, DNA methylation levels at the $L E P$ and $A D I P O Q$ gene promoters in blood were recently found to be lower in obese and insulin resistant adolescents [26]. Altogether these results suggest that $L E P$ and $A D I P O Q$ DNA methylation profiles might be involved in the pathology of obesity and cardiometabolic diseases. Nevertheless, LEP and ADIPOQ epigenetic profiles in adipose tissue and their associations with obesity and obesity-associated metabolic perturbations have not been assessed so far. Accordingly, we hypothesized that decreased $L E P$ DNA methylation and increased $A D I P O Q$ DNA methylation in adipose tissue could lead to higher degree of obesity and proinflammatory state, dyslipidemia, hypertension and insulin resistance. Henceforth, the objective of this study was to determine whether $L E P$ and ADIPOQ DNA methylation levels in subcutaneous (SAT) and visceral (VAT) adipose tissues were associated with obesity and obesity-related complications severely obese men and women. SAT and VAT were both analysed because they show specific gene expression profiles (ex. LEP and ADIPOQ) [27,28] and associations with cardiovascular risk factors [29]. Moreover, we tested whether adipokine epigenetic profiles in blood reflect those in adipose tissues and whether they could be used as proxies.

\section{Methods}

\section{Subjects}

Blood, SAT and VAT samples were obtained from 33 men and 40 premenopausal women $\left(B M I>40 \mathrm{~kg} / \mathrm{m}^{2}\right.$ ) undergoing bioliopancreatic diversion with duodenal switch to treat obesity. They were selected based on the fact that they were free of treatment for dyslipidemia, hypertension and diabetes. The surgical and sampling procedures have been described previously [30,31]. All participants provided a written informed consent before their inclusion in the study, and all clinical data were denominalized. This project was performed in collaboration with the Tissue bank for the study of obesity and its complications at the Institut Universitaire de Cardiologie et de Pneumologie de Québec. The project was approved by this institution's and the Université Laval's ethics committees and was conducted in accordance with the Declaration of Helsinki.

\section{Nucleic acid extraction}

DNA was purified from whole blood samples with the Gentra Puregene Blood Kit (Qiagen, Valencia, CA). DNA and RNA from SAT and VAT were extracted as previously described [32]. RNA quality was assessed with Agilent 2100 Bioanalyzer RNA Nano Chips (Agilent Technologies, Santa Clara, CA). Three RNA samples from SAT and VAT had low RNA integrity numbers $(\mathrm{RIN}<6.0)$ and were excluded from the analysis. The other RNA samples in SAT and VAT showed a high quality with mean RIN values of $8.0 \pm 0.8$ and $8.3 \pm 0.6$ respectively. RNA samples were not available for blood samples. 


\section{DNA methylation analyses and genotyping}

DNA methylation levels at CpG sites were assessed using pyrosequencing (Pyromark Q24, QIAGEN-Biotage). Combined with the NaBis DNA treatment, pyrosequencing is a quantitative real-time sequencing technology that allows to measure DNA methylation levels (\%) at a single cytosine $(\mathrm{CpG})$ of a given genomic region. The NaBis treatment of DNA (EpiTect Bisulfite Kit, Qiagen) specifically converts unmethylated cytosines into uracil, while the methylated cytosines are protected from this transition, creating a cytosine/thymine polymorphism. Once treated, NaBis-DNA is amplified (Pyromark PCR kit, Qiagen), and the cytosine and thymine alleles are quantified by pyrosequencing [33]. Specific PCR and pyrosequencing primer pairs for $L E P$ and $A D I P O Q$ DNA methylation analyses are described in Additional files 1 and 2.

Genotyping of the LEP single nucleotide polymorphism (SNP) rs2167670 was performed in the three tissues using pyrosequencing as reported before [34]. The rs2167270 genotype was identical in all three tissues analysed for the 73 patients. Genotype frequencies (GG: 30 (41.1\%); GA: $35(47.9 \%)$ and AA: $8(11.0 \%)$ ) were found to be under Hardy-Weinberg equilibrium $(p>0.05)$. Carriers of the minor A allele (GA/AA) were grouped together for statistical analysis purposes as the number of homozygous AA was very low.

\section{LEP and ADIPOQ mRNA measurements}

mRNA levels in SAT and VAT were quantified by quantitative real-time PCR (qRT-PCR). Complementary DNA (cDNA) was generated from total RNA using a random primer hexamer provided with the High Capacity cDNA Archive Kit from Applied Biosystems (Foster City, CA). Equal amounts of cDNA were run in duplicate and amplified in a $20 \mu \mathrm{L}$ reaction containing $10 \mu \mathrm{L}$ of Universal PCR Master Mix (Applied Biosystems). Primers and Taqman probes were obtained from Applied Biosystems (LEP: Hs00174877_m1 and ADIPOQ: Hs00605917_m1). The Glyceraldehyde 3-phosphate dehydrogenase (GAPDH: Hs99999905_m1; Applied Biosystems) housekeeping gene was amplified in parallel. LEP, ADIPOQ and GAPDH amplifications were performed using the Applied Biosystems 7500 Real Time PCR System, as recommended by the manufacturer (Applied Biosystems). LEP and ADIPOQ mRNA $(\mathrm{Ct})$ levels were quantified relative to change in GAPDH gene expression (Ct). GAPDH/ADIPOQ and $G A P D H / L E P C$ tratios $(1 / \mathrm{x})$ were used for the analysis.

\section{Statistical analyses}

The mean DNA methylation of the 21 CpGs analysed in the proximal promoter $\mathrm{CpG}$ island of $L E P$ gene (Additional file 3) was first computed (LEP-Mean) and analysed. Moreover, since we [34] and other groups [35-37] have previously reported that CpGs located between $\mathrm{CpG7}$ to
CpG17 are of first interest for $L E P$ gene expression regulation through DNA methylation changes, we analysed these CpGs individually to identify those more likely to be regulatory. Out of these $8 \mathrm{CpGs}$, we excluded the $\mathrm{CpG}$ sites that were found to be unmethylated $(\leq 10.0 \%)$ or hypermethylated $(\geq 90 \%)$ as they show low DNA methylation variability and are thus unlikely to explain the phenotypic variability. Hence, CpG12 to CpG16 were not analysed further in adipose tissues and blood (Additional file 3).

At $A D I P O Q$ gene locus, $\mathrm{CPG}$ island $\mathrm{A}$ and $\mathrm{C}$ were either unmethylated $(\leq 10.0 \%)$ or hypermethylated $(\geq 90 \%)$ and were thus not further analysed (Additional file 4). Mean DNA methylation levels were computed for CpG island $\mathrm{E}$ in SAT, VAT (ADIPOQ-Mean). CpGE1 and CPGE3 were analysed individually in SAT and VAT, whereas CpGE2 (hypermethylated) was not analysed further. In blood, CpGE3 was the only one analysed as the ADIPOQ-Mean, and CpGE1 and CpGE2 were found to be hypermethylated (Additional file 4).

The normal distribution of all variables was assessed using a Kolmogorov-Smirnov test. Fasting triglyceride (TG), C-reactive protein (CRP) and both fasting glucose and DNA methylation levels at ADIPOQ-CpGE3 locus in blood were found to be normally distributed after they were log10-transformed and ranked respectively. The associations between adipokine genes DNA methylation levels, anthropometric measures and metabolic profile were assessed with partial Pearson's correlations. The partial Pearson correlations were also used to determine the relationship between fasting low-density lipoprotein cholesterol (LDL-C) levels, LEP DNA methylation and mRNA levels in SAT. Pearson's correlation coefficients were adjusted for the following covariates when appropriate: sex, age, and waist circumference. Waist circumference is a stronger cardiometabolic disease risk marker and was preferred to BMI as a covariate $[38,39]$. Moreover, all results remained unchanged after consideration of smoking status. Two-sided $p$-values $\leq 0.05$ were considered statistically significant. The statistical analyses were performed with the IBM SPSS Statistics 20 software (release 20.0.0, SPSS, Chicago, Il, USA).

\section{Results}

The characteristics of the subjects included in this study are shown in Table 1. All subjects were severely obese (obese class III [40]) with BMI ranging from 40.0 to $60.0 \mathrm{~kg} / \mathrm{m}^{2}$. The patients were slightly hypertensive but had generally good metabolic health without diabetes or dyslipidemia $[41,42]$.

\section{LEP and ADIPOQ DNA methylation and anthropometric variables}

To determine whether $L E P$ and $A D I P O Q$ epigenetic profiles are involved in the pathogenesis of obesity and 
Table 1 Characteristics of the subjects studied $(n=73)$

\begin{tabular}{|c|c|c|}
\hline & Mean \pm SD & Range (min-max) \\
\hline Men (\%) & $33(45.2 \%)$ & - \\
\hline Age (years) & $34.7 \pm 7.1$ & $21.4-53.8$ \\
\hline BMI $\left(\mathrm{kg} / \mathrm{m}^{2}\right)$ & $49.6 \pm 6.0$ & $40.0-60.0$ \\
\hline Hip circumference (cm) & $147.7 \pm 14.0$ & $123.0-193.0$ \\
\hline Waist circumference (cm) & $139.3 \pm 16.6$ & $99.0-180.0$ \\
\hline Waist hip ratio & $0.95 \pm 0.11$ & $0.68-1.17$ \\
\hline Fasting glucose $\left(\mathrm{mmol} / \mathrm{l}^{\mathrm{a}}\right.$ & $5.51 \pm 1.05$ & $4.00-8.70$ \\
\hline Systolic blood pressure (mm Hg) & $137.0 \pm 14.0$ & $101.0-183.0$ \\
\hline Diastolic blood pressure (mm Hg) & $86.0 \pm 10.0$ & $57.0-108.0$ \\
\hline TC (mmol/l) & $4.87 \pm 0.85$ & $3.22-6.92$ \\
\hline LDL-C (mmol/l) & $2.96 \pm 0.76$ & $1.20-5.40$ \\
\hline $\mathrm{HDL}-\mathrm{C}(\mathrm{mmol} / \mathrm{l})$ & $1.17 \pm 0.27$ & $0.70-2.12$ \\
\hline TC/HDL-C & $4.32 \pm 1.24$ & $0.98-7.56$ \\
\hline $\mathrm{TG}^{\mathrm{a}}(\mathrm{mmol} / \mathrm{l})$ & $1.52 \pm 0.98$ & $0.52-6.89$ \\
\hline $\mathrm{CRP}^{\mathrm{a}}(\mathrm{mg} / \mathrm{L})(\mathrm{n}=53)$ & $8.2 \pm 10.2$ & $2.0-54.9$ \\
\hline Current smokers & $17(23.3 \%)$ & - \\
\hline
\end{tabular}

BMI, body Mass Index; TC, total cholesterol; LDL-C, low-density lipoprotein-cholesterol $\mathrm{HDL}-\mathrm{C}$, high-density lipoprotein-cholesterol; TC/HDL-C, total cholesterol to high-density lipoprotein-cholesterol ratio; TG, triglycerides; CRP, c-reactive protein. ${ }^{a}$ Geometric mean.

cardiometabolic complications, we first assessed the associations between $L E P$ and ADIPOQ DNA methylation levels in adipose tissues and blood and obesity-related anthropometric measures. The mean DNA methylation levels of $L E P$ promoter $\mathrm{CpG}$ island were found to be negatively correlated with BMI $(\mathrm{r}=-0.328 ; p=0.005)$ and hip circumference $(\mathrm{r}=-0.230 ; p=0.05)$ (Pearson's correlation coefficients adjusted for age and sex) (Additional file 5). To identify the CpGs that are more likely to be regulatory, LEP-CpG7, LEP-CpG11 and LEP-CpG17 were analysed independently. Negative correlations were observed between BMI and LEP-CpG7 ( $\mathrm{r}=-0.252 ; p=0.03)$ and LEP-CpG11 ( $\mathrm{r}=-0.234 ; p=0.05)$ (Figure 1-Panel A and B). As obesity-related proinflammatory response is associated with higher plasma leptin levels, we also assessed whether LEP DNA methylation levels in blood cells are associated with circulating levels of the pro-inflammatory CRP. Lower LEP-Mean and LEP-CpG7 DNA methylation levels in blood were associated with increased levels of CRP (LEP-Mean: $\mathrm{r}=-0.285 ; p=0.05$ and LEP-CpG7: $\mathrm{r}=-0.397, p=0.004, \mathrm{n}=53$ ) (Additional file 6). Of note, the correlations reported between LEP DNA methylation levels in blood and BMI were no longer significant after adjusting for CRP levels (LEP-Mean: $\mathrm{r}=-0.237$; $p=0.10 ; L E P$-CpG7: $\mathrm{r}=-0.151 ; p=0.294$ and LEPCpG11: $\mathrm{r}=-0.155 ; p=0.283(\mathrm{n}=53)$ ).

$A D I P O Q$ DNA methylation levels in SAT were found correlated with BMI (ADIPOQ-Mean: $\mathrm{r}=0.250 ; p=0.04$ and ADIPOQ-CpGE1: $\mathrm{r}=0.265 ; p=0.03$ ) (Figure 1-Panel C)
(Additional file 5) and waist circumference (ADIPOQ-Mean: $\mathrm{r}=0.361 ; p=0.002 ; A D I P O Q-C p G E 1: \mathrm{r}=0.364 ; p=0.002$ and $A D I P O Q-C p G E 3: \mathrm{r}=0.304 ; p=0.01$ ) (Figure 2-Panel $\mathrm{A}$ and $\mathrm{B}$ ) (Additional file 5). Moreover, DNA methylation levels at $A D I P O Q-C p G E 3$ locus in SAT were positively correlated with hip circumference $(\mathrm{r}=0.234 ; p=0.05)$ (Additional file 5). No additional association was observed between $L E P$ and $A D I P O Q$ DNA methylation levels and anthropometric variables (Additional file 5).

\section{LEP and ADIPOQ DNA methylation and obesity-related complications}

We next determined whether $L E P$ and $A D I P O Q$ DNA methylation levels in adipose tissue and blood were associated with obesity-related complications including dyslipidemia, hyperglycemia and hypertension. Fasting low-density lipoprotein-cholesterol (LDL-C) levels were positively correlated with $L E P$-Mean DNA methylation levels in VAT $(\mathrm{r}=0.273 ; p=0.02)$ and ADIPOQ-Mean DNA methylation levels in SAT $(\mathrm{r}=0.268 ; p=0.03)$ and VAT $(\mathrm{r}=0.245 ; p=$ 0.04) (Table 2). Analyses of individual CpG sites revealed positive correlations between LDL-C levels and DNA methylation levels at LEP-CpG17 locus in blood $(\mathrm{r}=$ 0.234; $p=0.05)$ and SAT $(\mathrm{r}=0.391 ; p=0.001)$ (Table 2). At the ADIPOQ gene loci, CpGE1 $(\mathrm{r}=0.236 ; p=0.05)$ and CpGE3 $(r=0.292 ; p=0.01)$ in SAT and CpGE1 in VAT (CpGE1: $\mathrm{r}=0.267 ; p=0.03$ ) were also positively correlated with fasting LDL-C levels (Table 2). A trend for association was also observed between LDL-C and DNA methylation levels at LEP-CpG11 in blood and SAT (Table 2). DNA methylation levels at the $A D I P O Q$ gene loci (ADIPOQMean and ADIPOQ-CpGE3) in SAT and at $L E P$-CpG7 in VAT were associated with fasting total cholesterol (TC) and high-density lipoprotein-cholesterol (HDL-C), respectively (Additional file 6). Adipokine genes DNA methylation levels were not found to be associated with fasting glucose levels and either systolic or diastolic blood pressure (Additional file 6).

\section{LEP and ADIPOQ DNA methylation and mRNA levels in SAT and VAT, and obesity-related complications}

To further explore the functionality of the associations reported in the two previous sections, we verified whether LEP and ADIPOQ gene expression and DNA methylation levels in SAT and VAT were correlated with phenotypic variability.

We have previously reported that LEP-CpG17 DNA methylation levels are negatively associated with $L E P$ mRNA levels in SAT, specifically in carriers of the rs2167270 A alleles (GA/AA genotypes; $r=-0.367, p=0.02$ ) [34] (Table 3). The $L E P$ genotypes were thus taken into account. LDL-C levels were found to be negatively correlated with $L E P$ mRNA levels $(\mathrm{r}=-0.317 ; p=0.04)$ in $\mathrm{rs} 2167270$ A allele carriers only (Table 3 ). Of note, the correlation between 


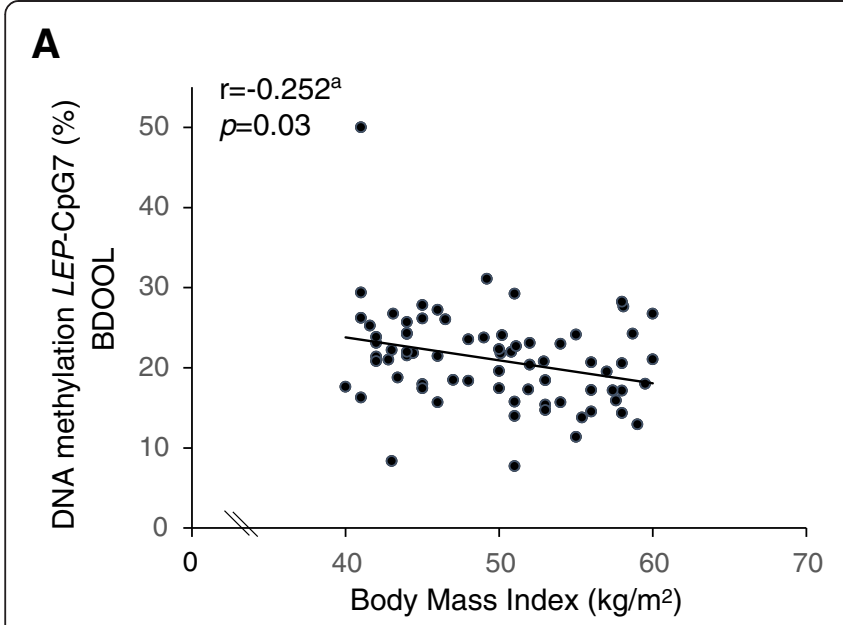

B

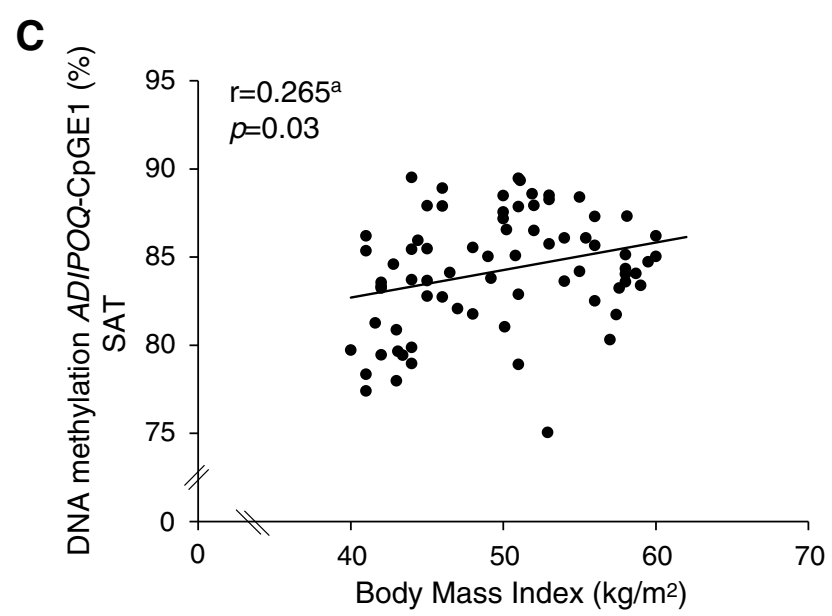

Figure 1 LEP and ADIPOQ DNA methylation levels according to body mass index (BMI) in severely obese patients. LEP DNA methylation levels at CpG7 (A) and CpG11 (B) in blood were associated with BMI. In SAT, ADIPOQ DNA methylation levels at CpGE1 were positively

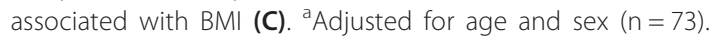

\section{A}

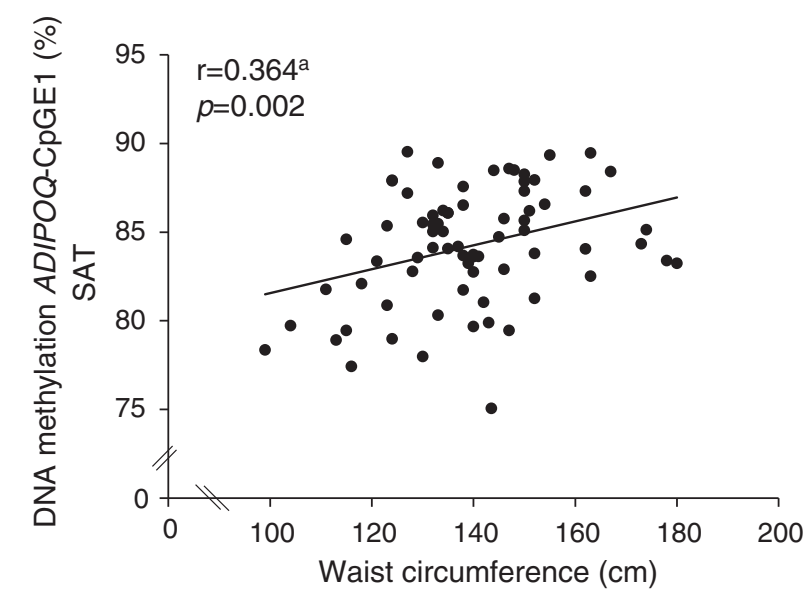

B

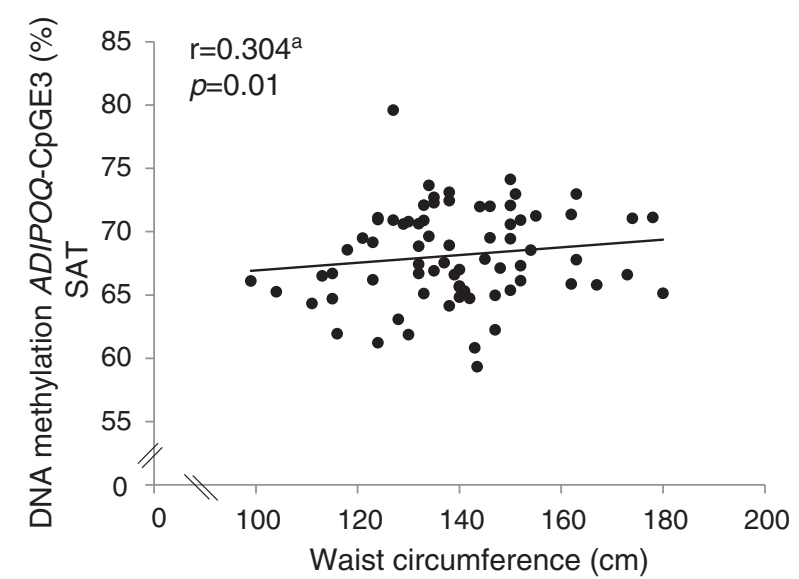

Figure 2 ADIPOQ DNA methylation levels according to waist circumference in severely obese patients. ADIPOQ DNA methylation levels at CpGE1

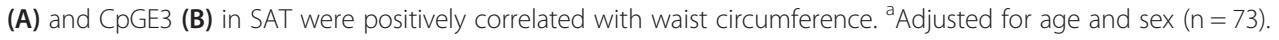


Table 2 Pearson correlation coefficients between fasting LDL-C levels and both LEP and ADIPOQ DNA methylation and mRNA levels in subcutaneous (SAT) and visceral adipose tissues (VAT)

\begin{tabular}{|c|c|c|c|c|}
\hline & \multicolumn{2}{|c|}{ LDL-C levels (mmol/L) Adjusted for age and sex } & \multicolumn{2}{|c|}{ LDL-C levels (mmol/L) Adjusted for age, sex and WG } \\
\hline & $r$ & $P$ & $r$ & $p$ \\
\hline \multicolumn{5}{|l|}{ BLOOD } \\
\hline LEP-CpG7 & 0.133 & 0.27 & 0.122 & 0.31 \\
\hline LEP-CpG11 & 0.222 & 0.06 & 0.206 & 0.09 \\
\hline LEP-CpG17 & 0.239 & 0.04 & 0.234 & 0.05 \\
\hline LEP-Mean & 0.178 & 0.138 & 0.162 & 0.18 \\
\hline$A D I P O Q-C p G E 3^{a}$ & 0.161 & 0.17 & 0.149 & 0.22 \\
\hline \multicolumn{5}{|l|}{ SAT } \\
\hline LEP-CpG7 & 0.090 & 0.46 & 0.108 & 0.37 \\
\hline LEP-CpG11 & 0.215 & 0.07 & 0.231 & 0.06 \\
\hline LEP-CpG17 & 0.380 & 0.001 & 0.391 & 0.001 \\
\hline LEP-Mean & 0.190 & 0.11 & 0.218 & 0.07 \\
\hline LEP mRNA levels & -0.113 & 0.35 & -0.098 & 0.42 \\
\hline ADIPOQ-CpGE1 & 0.176 & 0.14 & 0.236 & 0.05 \\
\hline ADIPOQ-CPGE3 & 0.242 & 0.04 & 0.292 & 0.01 \\
\hline ADIPOQ-Mean & 0.206 & 0.08 & 0.268 & 0.03 \\
\hline ADIPOQ mRNA levels & 0.024 & 0.85 & 0.009 & 0.94 \\
\hline \multicolumn{5}{|l|}{ VAT } \\
\hline LEP-CpG7 & 0.130 & 0.28 & 0.131 & 0.28 \\
\hline LEP-CpG11 & 0.158 & 0.19 & 0.169 & 0.16 \\
\hline LEP-CpG17 & 0.114 & 0.34 & 0.127 & 0.30 \\
\hline LEP-Mean & 0.256 & 0.03 & 0.273 & 0.02 \\
\hline LEP mRNA levels & 0.160 & 0.18 & 0.034 & 0.78 \\
\hline ADIPOQ-CPGEI & 0.270 & 0.02 & 0.267 & 0.03 \\
\hline ADIPOQ-CPGE3 & 0.188 & 0.12 & 0.181 & 0.14 \\
\hline ADIPOQ-Mean & 0.250 & 0.04 & 0.245 & 0.04 \\
\hline ADIPOQ mRNA levels & -0.111 & 0.37 & -0.121 & 0.33 \\
\hline
\end{tabular}

WG,waist girth.

${ }^{a}$ Results obtained after rank transformation of DNA methylation levels.

Values in bold type are statistically significant: $p \leq 0.05$.

LEP mRNA and LDL-C levels was no longer significant after adjusting Pearson's correlation coefficient for LEPCpG17 DNA methylation levels $(\mathrm{r}=-0.205 ; p=0.21)$ (Table 3).

In SAT, DNA methylation levels at ADIPOQ-CpGE3 were negatively correlated with $A D I P O Q$ mRNA levels $(\mathrm{r}=0.257 ; p=0.03)$. ADIPOQ mRNA levels in SAT or VAT were not associated with BMI, waist girth, hip circumference or LDL-C levels (Table 2 and Additional file 5).

\section{Discussion}

In the current study, we report that LEP and ADIPOQ DNA methylation levels, measured in paired adipose tissues and blood samples, are associated with obesity-related anthropometric variables and fasting LDL-C levels.
We first demonstrated that lower LEP promoter DNA methylation levels in blood cells are associated with higher BMI. Several cross-sectional studies have reported lower LEP DNA methylation levels in blood cells of newborns and children with higher birth weight and BMI [25], in obese and insulin resistant adolescents [26] and obese women [43]. Our findings are also concordant with fetal metabolic programming studies in humans and rodent models which have lately shown that lower LEP DNA methylation levels in blood, placenta, VAT, liver and muscle are associated with a risk for obesity and metabolic diseases in the offspring $[10,44,23]$. It is thus tempting to speculate on whether the LEP epigenetic profile we are reporting in blood was established in utero and might have been involved in the development of obesity in the 
Table 3 Pearson correlation coefficients between low-density lipoprotein cholesterol (LDL-C) levels, LEP mRNA and LEP gene CpG17 DNA methylation levels in subcutaneous adipose tissue (SAT) according to the rs2167270 genotype

\begin{tabular}{|c|c|c|c|c|c|c|}
\hline & \multicolumn{3}{|c|}{ LEP mRNA levels } & \multicolumn{3}{|c|}{ LEP-CpG17 DNA methylation levels } \\
\hline & ALL & GG & GA/AA & ALL & GG & GA/AA \\
\hline & $n=73$ & $\mathrm{n}=\mathbf{3 0}$ & $n=43$ & $\mathrm{n}=73$ & $\mathrm{n}=30$ & $n=43$ \\
\hline LDL-C levels & -0.097 & 0.142 & $-0.317^{*}$ & $0.370^{* *}$ & $0.400^{*}$ & $0.384^{* *}$ \\
\hline \multicolumn{7}{|l|}{ Adjusted for sex and waist girth } \\
\hline LDL-C levels & -0.035 & 0.138 & -0.205 & - & - & - \\
\hline \multicolumn{7}{|c|}{ Adjusted for sex, waist girth and LEP-CpG17 DNA methylation levels } \\
\hline LDL-C levels & - & - & - & $0.360^{* *}$ & $0.399^{*}$ & $0.303^{+}$ \\
\hline \multicolumn{7}{|c|}{ Adjusted for sex, waist girth and LEP mRNA levels } \\
\hline LEP-CpG17 DNA methylation levels & -0.177 & 0.038 & $-0.367^{*}$ & - & - & - \\
\hline \multicolumn{7}{|l|}{ Adjusted for sex } \\
\hline LEP-CpG17 DNA methylation levels & -0.152 & -0.021 & $-0.282^{\dagger}$ & - & - & - \\
\hline Adjusted for sex and LDL-C levels & & & & & & \\
\hline
\end{tabular}

Pearson correlations are statistically significant: ${ }^{+} p \leq 0.10 ;{ }^{*} p \leq 0.05 ;{ }^{* *} p \leq 0.01$.

patients of the current study. However, we would have expected that the LEP epigenetic signature that we are describing in blood would also have been present in adipose tissues, if programmed in utero. Because the correlations between LEP DNA methylation levels and BMI were attenuated after adjusting for CRP levels, we hypothesized that the obesity-related proinflammatory response per se contributed to the alteration of LEP epigenetic profile in blood cells. Leptin is mainly produced by adipose tissues but is also expressed by peripheral blood mononuclear cells [45]. It is also recognized that plasma leptin and CRP levels are upregulated in obesity and proinflammatory states and closely related [46]. Plasmatic elevation of CRP levels observed in obese subjects has been associated with higher plasma concentrations and leptin resistance [46]. Hence, increased CRP levels might, to some extent, regulate plasmatic leptin concentrations and leptin resistance via the modification of LEP DNA methylation levels. Unfortunately, because blood cell counts were not available, we cannot exclude that LEP DNA methylation variability and the associations reported with CRP and BMI might be partially attributed to blood cell composition changes between samples. However, if cellular composition changes impact the results, this effect is likely modest as highlighted by Talens et al. [47]. DNA methylation regulation is (partly) tissue-specific and might explain the discrepancy between the associations reported in blood and adipose tissues $[48,49]$. Also, we cannot exclude that LEP epigenetic profile of these "metabolically-healthy" individuals might have been re-programmed by the post-natal environmental and stochastic factors.

We also report that $A D I P O Q$ DNA methylation levels in SAT are positively associated with BMI and waist circumference. ADIPOQ DNA methylation levels at CpGE3 are also associated with $A D I P O Q$ mRNA levels in SAT.
This suggests that epigenetic modifications at ADIPOQ gene locus are functional and could potentially be involved in the pathogenesis of impaired glucose tolerance and insulin resistance associated with obesity. Nevertheless, we cannot exclude that higher $A D I P O Q$ DNA methylation levels would have led to higher degree of obesity. In accordance with this hypothesis, we have lately reported that placental ADIPOQ DNA methylation levels are impaired following exposure to maternal glucose $2 \mathrm{~h}$ postOGTT at second trimester of pregnancy suggesting that $A D I P O Q$ epigenetic profile can increase susceptibility to obesity in the newborn [24]. Whether ADIPOQ epigenetic profile in SAT is established before or after the onset of obesity will need to be confirmed, but is clearly of strong interest.

Interestingly, LEP DNA methylation levels in blood, SAT and VAT, and ADIPOQ DNA methylation levels in SAT and VAT were both found to be positively associated with LDL-C levels suggesting a common epigenetic regulation independently of their biological roles although these two adipokines are oppositely regulated. In addition, we report that the association between LDL-C and $L E P$ mRNA levels are partially dependent on $L E P$ rs2167270 genotype and DNA methylation levels at CpG17. In vitro studies have shown that the incubation of endothelial cells with LDL or oxidized-LDL increases DNA methylation levels at specific gene loci through the induction of DNA methyltransferase 1 (DNMT1) expression and activity $[50,51]$. Interestingly, the use of statins, the most prescribed lipid lowering drugs, is associated with the down regulation of DNMT activity and demethylation of $B M P 2$ promoter in mice and cell culture models [52]. Whether the effect of statins is direct (DNMT activity) or mediated through LDL-C lowering needs to be further investigated. Still, our results and those from these previous studies 
suggest that LDL-C might be involved in the regulation of $L E P$ and $A D I P O Q$ gene DNA methylation levels with possible impacts on mRNA transcription, and that, independently of their biological roles. It is recognized that the treatment of dyslipidemia with statins increases insulin resistance and the risk for type 2 diabetes $[53,54]$. The modifications of LDL-C levels and adipokines epigenetic profile by statins might be one of the mechanisms contributing to the insulin resistance state. Consequently, it will be of great clinical interest to confirm the directionality of these associations and to further investigate their respective mechanisms of regulation through epigenetic changes.

The identification of DNA methylation surrogate measures in readily accessible tissue remains an important challenge in epigenetic epidemiology $[55,11]$. In the current study, paired adipose tissues and blood samples were analysed and tested to assess whether they show DNA methylation profile similarities at adipokine gene loci. DNA methylation levels at LEP-CpG11 and CpG17 were found to be associated with LDL-C levels in both SAT and blood samples. These two CpGs were recently identified by our group among the most promising $\mathrm{CpG}$ sites to be used in blood as a surrogate for LEP DNA methylation in adipose tissues [34]. DNA methylation levels at CpG11 and CpG17 were found to be modestly but significantly correlated between SAT and blood $(r=0.43, p<0.01 ; r=0.58$, $\mathrm{p}<0.01$ respectively) [34]. These two CpGs are respectively within $C / E B P$ and $S P 1$ transcription factor binding sites (TFBS), both involved in $L E P$ gene expression regulation $[56,35]$. The correlations reported between LEPCpG11 and -CpG17 DNA methylation and LDL-C levels in SAT and blood suggesting that DNA methylation regulation by LDL-C at these two CpG sites is common to both tissues. Moreover, as these associations are similar in SAT and blood, they are very likely independent of cellular count. The current results thus support that LEPCpG11 and -CpG17 DNA methylation levels in blood are potential relevant surrogates for SAT DNA methylation levels.

The strengths of the current study include analyses of two adipokines central in energy metabolism regulation in two adipose tissue compartments and in blood. Blood is the most clinically accessible tissue whereas adipose tissues are those biologically relevant for the study of obesity and adipokine epigenetic regulation. Access to adipose tissues remains restricted, and this study provides insights on the use of blood as a potential surrogate tissue in epigenetic epidemiology. None of our participants were taking medication to treat any of the metabolic syndrome components and were on average metabolically fit. This metabolically-healthy obese population has a unique obesity phenotype and metabolism, and $L E P$ and $A D I P O Q$ DNA methylation profiles might be distinct in these participants. Consequently, the results reported in the current study need to be validated in normal weight populations as well as obese populations with cardiometabolic complications. Moreover, as we are reporting crosssectional observations, longitudinal and functional studies are needed to determine the causality of the relationships between adipokine DNA methylation, anthropometric variables and plasma LDL-C levels.

\section{Conclusions}

This study provides further evidence that $L E P$ DNA methylation levels in blood cells and $A D I P O Q$ DNA methylation levels in SAT are associated with obesity-related anthropometric measures. It also suggests that LDL-C, known to regulate DNA methylation processes, could be involved in adipokines' gene expression regulation through epigenetic changes. Interestingly, similar correlations were observed between LEP DNA methylation and LDL-C levels in blood and SAT. This might be of clinical importance considering that the access to biologically active tissues, such as adipose tissues is limited.

\section{Additional files}

\begin{abstract}
Additional file 1: PCR and pyrosequencing primers for LEP gene proximal promoter $C p G$ island amplification and pyrosequencing. Additional file 2: PCR and pyrosequencing primers for ADIPOQ gene CpG islands amplification and pyrosequencing.

Additional file 3: CpG island within LEP gene proximal promoter region. The CpG sites are numbered according to Bouchard et al. 2010 [23]. The underlined CpG sites were epigenotyped in the current study, Associations between LEP DNA methylation levels, anthropometric variables and obesity-related complications were assessed at CpG sites for which DNA methylation values are in bold type. The sequence $(-11131$ to -10563$)$ was numbered relative to the first leptin AUG codon (UCSC Genome Browser (Human Feb. 2009)).
\end{abstract}

Additional file 4: $\mathrm{CpG}$ islands analysed within $A D I P O Q$ gene locus. CpG islands $A$ and $C$ were found to be hypomethylated and hypermethylated respectively and were not analysed. Associations between ADIPOQ DNA methylation levels, anthropometric variables and obesity-related complications were assessed at CpG sites for which DNA methylation values are in bold type.

Additional file 5: Pearson correlation coefficients between LEP and ADIPOQ DNA methylation and mRNA levels in blood, subcutaneous (SAT) and visceral adipose tissue (VAT) and anthropometric variables (adjusted for age and sex) $(n=73)$.

Additional file 6: Pearson correlation coefficients between LEP and ADIPOQ DNA methylation and mRNA levels in blood, subcutaneous (SAT) and visceral adipose tissues (VAT) and cardiometabolic risk factors (adjusted for age, sex and waist circumference) $(n=73)$.

\section{Abbreviations}

ADIPOQ: Adiponectin; BMI: Body mass index; CRP: C-reactive protein; DNMT: DNA methyltransferase; HDL-C: High-density lipoprotein cholesterol; LDL-C: Low-density lipoprotein cholesterol; LEP: Leptin; SAT: Subcutaneous adipose tissue; SNP: Single nucleotide polymorphism; TC: Total cholesterol; VAT: Visceral adipose tissue.

\section{Competing interests}

The authors declare that they have no competing interests. 


\section{Authors' contributions}

$\mathrm{AAH}$ contributed to the study design, performed the data collection, the data analysis/interpretation and wrote the manuscript. $C L$ contributed to data collection and revised the manuscript. SB, OL, L.Biertho, SM, MCV and AT participated in the conception of the study design and revised the manuscript. MFH and L.Bouchard conceived the study design, participated in the data analysis/interpretation process and revised the manuscript. All authors read and approved the final manuscript.

\section{Acknowledgments}

The Authors acknowledge the invaluable collaboration of the surgery team, bariatric surgeons (Hould F.S., Lebel S., Marceau P.) and biobank staff of the IUCPQ. The authors also express their gratitude to Céline Bélanger, Chicoutimi Hospital, for her thoughtful language revision of the manuscript. L.Bouchard is a junior research scholar from the Fonds de la recherche du Québec - santé (FRQS). M.F.H. was a FRSQ Research Scholar and was awarded Clinical Scientist award by the Canadian Diabetes Association; she is now supported by American Heart Association (AHA) and American Diabetes Association (ADA) awards. L.Bouchard and M.F.H. are members of the FRQS-funded Centre de recherche du CHUS. A.T. is the director of a Research Chair in Bariatric and Metabolic Surgery. M.C.V. is the recipient of the Canada Research Chair in Genomics Applied to Nutrition and Health. A.A.H. is the recipient of a doctoral training award from the FRQS and the Faculte de Médecine et des Sciences de la Santé de I'Université de Sherbrooke. This project was supported by the Canadian Institutes of Health Research and the FRQS.

\section{Author details}

'Department of Biochemistry, Université de Sherbrooke, Sherbrooke, QC, Canada. ${ }^{2}$ ECOGENE-21 and Clinical Research Center and Lipid Clinic, Chicoutimi Hospital, Saguenay, QC, Canada. ${ }^{3}$ Quebec Heart and Lung Institute, Quebec, Canada. ${ }^{4}$ Department of Surgery, Laval University, Quebec, Canada. ${ }^{5}$ Axe Endocrinologie et Néphrologie, Centre de recherche du CHU de Québec, Quebec, QC, Canada. 'Department of Food Science and Nutrition, Laval University, Québec, QC, Canada. ${ }^{7}$ Institute of Nutrition \& Functional Foods, Université Laval, Quebec, QC, Canada. ${ }^{8}$ Department of Medicine, Division of Endocrinology, Université de Sherbrooke, Sherbrooke, QC, Canada. ${ }^{9}$ Department of Population Medicine, Harvard Pilgrim Health Care Institute, Boston, MA, USA. ${ }^{10}$ General Medicine Division, Massachusetts General Hospital, Boston, MA, USA.

Received: 12 January 2015 Accepted: 22 April 2015 Published online: 01 May 2015

\section{References}

1. Malik VS, Willett WC, Hu FB. Global obesity: trends, risk factors and policy implications. Nat Rev Endocrinol. 2013;9(1):13-27. doi:10.1038/nrendo.2012.199.

2. Swinburn BA, Sacks G, Hall KD, McPherson K, Finegood DT, Moodie ML, et al. The global obesity pandemic: shaped by global drivers and local environments. Lancet. 2011;378(9793):804-14. doi:10.1016/S0140-6736(11)60813-1.

3. Yang W, Kelly T, He J. Genetic epidemiology of obesity. Epidemiol Rev. 2007;29:49-61. doi:10.1093/epirev/mxm004.

4. Waalen J. The genetics of human obesity. Translational research: the journal of laboratory and clinical medicine. 2014. doi:10.1016/j.trsl.2014.05.010.

5. Hebebrand J, Volckmar AL, Knoll N, Hinney A. Chipping away the 'missing heritability': GIANT steps forward in the molecular elucidation of obesity - but still lots to go. Obes Facts. 2010;3(5):294-303. doi:10.1159/000321537.

6. Jaenisch R, Bird A. Epigenetic regulation of gene expression: how the genome integrates intrinsic and environmental signals. Nat Genet. 2003;33:245-54. doi:10.1038/ng1089 ng1089.

7. Turner BM. Epigenetic responses to environmental change and their evolutionary implications. Philos Trans R Soc Lond B Biol Sci. 2009;364(1534):3403-18. doi:10.1098/rstb.2009.0125.

8. Henikoff S, Matzke MA. Exploring and explaining epigenetic effects. Trends Genet. 1997;13(8):293-5. doi:S0168952597012195.

9. Bird A. DNA methylation patterns and epigenetic memory. Genes Dev. 2002;16(1):6-21. doi:10.1101/gad.947102.

10. Tobi EW, Lumey LH, Talens RP, Kremer D, Putter H, Stein AD, et al. DNA methylation differences after exposure to prenatal famine are common and timing- and sex-specific. Hum Mol Genet. 2009;18(21):4046-53. doi:10.1093/hmg/ddp353.
11. Houde AA, Hivert MF, Bouchard L. Fetal epigenetic programming of adipokines. Adipocyte. 2013;2(1):41-6. doi:10.4161/adip.22055.

12. Pilsner JR, Hall MN, Liu X, llievski V, Slavkovich V, Levy D, et al. Influence of prenatal arsenic exposure and newborn sex on global methylation of cord blood DNA. PloS One. 2012;7(5), e37147. doi:10.1371/journal.pone.0037147.

13. Ling C, Ronn T. Epigenetic adaptation to regular exercise in humans. Drug Discov Today. 2014. doi:10.1016/j.drudis.2014.03.006.

14. Gillberg $L$, Jacobsen SC, Ronn T, Brons C, Vaag A. PPARGC1A DNA methylation in subcutaneous adipose tissue in low birth weight subjects-impact of 5 days of high-fat overfeeding. Metabolism. 2014;63(2):263-71. doi:10.1016/j.metabol.2013.10.003.

15. Dick KJ, Nelson CP, Tsaprouni L, Sandling JK, Aissi D, Wahl S et al. DNA methylation and body-mass index: a genome-wide analysis. Lancet. 2014. doi:10.1016/S0140-6736(13)62674-4.

16. Milagro Fl, Gomez-Abellan P, Campion J, Martinez JA, Ordovas JM, Garaulet M. CLOCK, PER2 and BMAL1 DNA methylation: association with obesity and metabolic syndrome characteristics and monounsaturated fat intake. Chronobiol Int. 2012;29(9):1180-94. doi:10.3109/07420528.2012.719967.

17. Jacobsen SC, Gillberg L, Bork-Jensen J, Ribel-Madsen R, Lara E, Calvanese V et al. Young men with low birthweight exhibit decreased plasticity of genome-wide muscle DNA methylation by high-fat overfeeding. Diabetologia. 2014:57(6):1154-8. doi:10.1007/s00125-014-3198-8.

18. Bouchard L, Rabasa-Lhoret R, Faraj M, Lavoie ME, Mill J, Perusse L, et al. Differential epigenomic and transcriptomic responses in subcutaneous adipose tissue between low and high responders to caloric restriction. Am J Clin Nutr. 2010;91(2):309-20. doi:10.3945/ajen.2009.28085.

19. Ronn T, Volkov P, Davegardh C, Dayeh T, Hall E, Olsson AH, et al. A six months exercise intervention influences the genome-wide DNA methylation pattern in human adipose tissue. PLoS Genet. 2013;9(6), e1003572. doi:10.1371/journal.pgen.1003572.

20. Moleres A, Campion J, Milagro Fl, Marcos A, Campoy C, Garagorri JM, et al. Differential DNA methylation patterns between high and low responders to a weight loss intervention in overweight or obese adolescents: the EVASYON study. FASEB J. 2013;27(6):2504-12. doi:10.1096/fj.12-215566.

21. Fantuzzi G. Adipose tissue, adipokines, and inflammation. J Allergy Clin Immunol. 2005;115(5):911-9. quiz 20. doi:10.1016/j.jaci.2005.02.023.

22. Havel PJ. Update on adipocyte hormones: regulation of energy balance and carbohydrate/lipid metabolism. Diabetes. 2004;53 Suppl 1:S143-51.

23. Bouchard L, Thibault S, Guay SP, Santure M, Monpetit A, St-Pierre J, et al. Leptin gene epigenetic adaptation to impaired glucose metabolism during pregnancy. Diab Care. 2010;33(11):2436-41. doi:10.2337/dc10-1024.

24. Bouchard L, Hivert MF, Guay SP, St-Pierre J, Perron P, Brisson D. Placental adiponectin gene DNA methylation levels are associated with mothers' blood glucose concentration. Diabetes. 2012;61(5):1272-80. doi:10.2337/db11-1160.

25. Obermann-Borst SA, Eilers PH, Tobi EW, de Jong FH, Slagboom PE, Heijmans BT, et al. Duration of breastfeeding and gender are associated with methylation of the LEPTIN gene in very young children. Pediatr Res. 2013;74(3):344-9. doi:10.1038/pr.2013.95

26. Garcia-Cardona MC, Huang F, Garcia-Vivas JM, Lopez-Camarillo C, Del Rio Navarro BE, Navarro Olivos E et al. DNA methylation of leptin and adiponectin promoters in children is reduced by the combined presence of obesity and insulin resistance. Int J Obes (Lond). 2014. doi:10.1038/ijo.2014.30.

27. van Harmelen $V$, Dicker $A$, Ryden $M$, Hauner $H$, Lonnqvist $F$, Naslund $E$, et al. Increased lipolysis and decreased leptin production by human omental as compared with subcutaneous preadipocytes. Diabetes. 2002;51(7):2029-36.

28. Lihn AS, Bruun JM, He G, Pedersen SB, Jensen PF, Richelsen B. Lower expression of adiponectin mRNA in visceral adipose tissue in lean and obese subjects. Mol Cell Endocrinol. 2004;219(1-2):9-15. doi:10.1016/j.mce.2004.03.002.

29. Neeland IJ, Ayers CR, Rohatgi AK, Turer AT, Berry JD, Das SR, et al. Associations of visceral and abdominal subcutaneous adipose tissue with markers of cardiac and metabolic risk in obese adults. Obesity (Silver Spring). 2013;21(9):E439-47. doi:10.1002/oby.20135.

30. Marceau P, Hould FS, Simard S, Lebel S, Bourque RA, Potvin M, et al. Biliopancreatic diversion with duodenal switch. World J Surg. 1998:22(9):947-54

31. Vohl MC, Sladek R, Robitaille J, Gurd S, Marceau P, Richard D, et al. A survey of genes differentially expressed in subcutaneous and visceral adipose tissue in men. Obes Res. 2004;12(8):1217-22. doi:10.1038/oby.2004.153.

32. Bouchard L, Faucher G, Tchernof A, Deshaies Y, Lebel S, Hould FS, et al. Comprehensive genetic analysis of the dipeptidyl peptidase-4 gene and 
cardiovascular disease risk factors in obese individuals. Acta Diabetol. 2009:46(1):13-21. doi:10.1007/s00592-008-0049-4

33. Dejeux E, El abdalaoui H, Gut IG, Tost J. Identification and quantification of differentially methylated loci by the pyrosequencing technology. Methods Mol Biol. 2009;507:189-205. doi:10.1007/978-1-59745-522-0_15.

34. Houde AA, Legare C, Hould FS, Lebel S, Marceau P, Tchernof A, et al. Cross-tissue comparisons of leptin and adiponectin: DNA methylation profiles. Adipocyte. 2014;3(2):132-40. doi:10.4161/adip.28308.

35. Melzner I, Scott V, Dorsch K, Fischer P, Wabitsch M, Bruderlein S, et al. Leptin gene expression in human preadipocytes is switched on by maturation-induced demethylation of distinct CpGs in its proximal promoter. J Biol Chem. 2002;277(47):45420-7. doi:10.1074/jbc.M208511200.

36. Stoger R. In vivo methylation patterns of the leptin promoter in human and mouse. Epigenetics. 2006;1 (4):155-62.

37. Marchi M, Lisi S, Curcio M, Barbuti S, Piaggi P, Ceccarini G, et al. Human leptin tissue distribution, but not weight loss-dependent change in expression, is associated with methylation of its promoter. Epigenetics. 2011;6(10):1198-206. doi:10.4161/epi.6.10.16600.

38. Janssen I, Katzmarzyk PT, Ross R. Waist circumference and not body mass index explains obesity-related health risk. Am J Clin Nutr. 2004;79(3):379-84.

39. Despres JP, Lemieux I, Bergeron J, Pibarot P, Mathieu P, Larose E, et al. Abdominal obesity and the metabolic syndrome: contribution to global cardiometabolic risk. Arterioscler Thromb Vasc Biol. 2008;28(6):1039-49. doi:10.1161/ATVBAHA.107.159228.

40. World Health Organisation. Obesity: preventing and managing the global epidemic. Report of a WHO consultation. World Health Organ Tech Rep Ser. 2000;894:i-xii, 1-253.

41. American Diabetes Association. Standards of medical care in diabetes-2014. Diab Care. 2014;37(1):S14-80. doi:10.2337/dc14-S014.

42. National Cholesterol Education Program Expert Panel. Third Report of the National Cholesterol Education Program (NCEP) Expert Panel on Detection, Evaluation, and Treatment of High Blood Cholesterol in Adults (Adult Treatment Panel III) final report. Circulation. 2002;106(25):3143-421.

43. Lesseur C, Armstrong DA, Paquette AG, Koestler DC, Padbury JF, Marsit CJ. Tissue-specific Leptin promoter DNA methylation is associated with maternal and infant perinatal factors. Mol Cell Endocrinol. 2013;381(1-2):160-7. doi:10.1016/j.mce.2013.07.024.

44. Khalyfa A, Carreras A, Hakim F, Cunningham JM, Wang Y, Gozal D. Effects of late gestational high-fat diet on body weight, metabolic regulation and adipokine expression in offspring. Int J Obes (Lond). 2013;37(11):1481-9. doi:10.1038/ijo.2013.12.

45. Samara A, Marie B, Pfister M, Visvikis-Siest S. Leptin expression in Peripheral Blood Mononuclear Cells (PBMCs) is related with blood pressure variability. Clin Chim Acta. 2008;395(1-2):47-50. doi:10.1016/j.cca.2008.04.028.

46. Hribal ML, Fiorentino TV, Sesti G. Role of $C$ reactive protein (CRP) in leptin resistance. Curr Pharm Des. 2014;20(4):609-15.

47. Talens RP, Boomsma DI, Tobi EW, Kremer D, Jukema JW, Willemsen G, et al Variation, patterns, and temporal stability of DNA methylation: considerations for epigenetic epidemiology. FASEB J. 2010;24(9):3135-44. doi:10.1096/fj.09-150490.

48. Song F, Smith JF, Kimura MT, Morrow AD, Matsuyama T, Nagase $H$, et al. Association of tissue-specific differentially methylated regions (TDMs) with differential gene expression. Proc Natl Acad Sci U S A. 2005;102(9):3336-41. doi:10.1073/pnas.0408436102.

49. Kitamura E, Igarashi J, Morohashi A, Hida N, Oinuma T, Nemoto N, et al. Analysis of tissue-specific differentially methylated regions (TDMs) in humans. Genomics. 2007;89(3):326-37. doi:10.1016/j.ygeno.2006.11.006.

50. Kumar A, Kumar S, Vikram A, Hoffman TA, Naqvi A, Lewarchik CM, et al. Histone and DNA methylation-mediated epigenetic downregulation of endothelial Kruppel-like factor 2 by low-density lipoprotein cholesterol. Arterioscler Thromb Vasc Biol. 2013;33(8):1936-42. doi:10.1161/ATVBAHA.113.301765.

51. Yang TC, Chen YJ, Chang SF, Chen CH, Chang PY, Lu SC. Malondialdehyde mediates oxidized LDL-induced coronary toxicity through the Akt-FGF2 pathway via DNA methylation. J Biomed Sci. 2014;21:11. doi:10.1186/1423-0127-21-11.

52. Kodach LL, Jacobs RJ, Voorneveld PW, Wildenberg ME, Verspaget HW, van Wezel T, et al. Statins augment the chemosensitivity of colorectal cancer cells inducing epigenetic reprogramming and reducing colorectal cancer cell 'stemness' via the bone morphogenetic protein pathway. Gut. 2011;60(11):1544-53. doi:10.1136/gut.2011.237495.
53. Sattar N, Preiss D, Murray HM, Welsh P, Buckley BM, de Craen AJ, et al. Statins and risk of incident diabetes: a collaborative meta-analysis of randomised statin trials. Lancet. 2010;375(9716):735-42. doi:10.1016/S0140-6736(09)61965-6.

54. Aiman U, Najmi A, Khan RA. Statin induced diabetes and its clinical implications. J Pharmacol Pharmacother. 2014;5(3):181-5. doi:10.4103/0976-500X.136097.

55. Mill J, Heijmans BT. From promises to practical strategies in epigenetic epidemiology. Nat Rev Genet. 2013;14(8):585-94. doi:10.1038/nrg3405.

56. Mason MM, He Y, Chen H, Quon MJ, Reitman M. Regulation of leptin promoter function by Sp1, C/EBP, and a novel factor. Endocrinology. 1998;139(3):1013-22. doi:10.1210/endo.139.3.5792.

\section{Submit your next manuscript to BioMed Central and take full advantage of:}

- Convenient online submission

- Thorough peer review

- No space constraints or color figure charges

- Immediate publication on acceptance

- Inclusion in PubMed, CAS, Scopus and Google Scholar

- Research which is freely available for redistribution 